\title{
Cryo-TEM Characterization of the Early Stages of the Uranium Oxalate Growth Evolution
}

\author{
Karen Kruska ${ }^{1}$, Shalini Tripathi ${ }^{1}$, Gabriel Hall ${ }^{1}$ and Edgar Buck ${ }^{2}$ \\ ${ }^{1}$ PNNL, United States, ${ }^{2}$ Pacific North West National Laboratory, United States
}

Owing to its curiously low solubility in acidic solutions, the oxalate anion has long been recognized as an excellent precipitating agent for trivalent lanthanides and actinides [1]. It is well established that f-element oxalates precipitate as agglomerates and are preserved in the oxide phase following calcination [2]. Multiple distinct morphologies are possible depending on the solution formation conditions. It is hypothesized that it is possible to predict an f-element oxide morphology based on its formation conditions. Furthermore, the formation conditions could be deduced from the observed oxide agglomerate morphology. Analysis of the particle nucleation and growth with in-situ electron microscopy is an effective method for probing the particle formation dynamics and therefore has been performed on many other chemical systems [3, 4]. As f-element oxalates are subject to rapid nucleation and growth, cryo transmission electron microscopy (TEM) was employed to freeze and capture those early stages of formation that result from the reaction of the f-metal nitrate and oxalic acid. The full growth cycle was investigated using a combination of optical and electron microscopies.

U nitrite was reacted with oxalic acid. Both optical microscopy (OM) and scanning electron microscopy (SEM) were used to observe the growth of oxalates in-situ. The OM used was a Nikon 600MEL metallurgical microscope with a Linkham thermal stage and the SEM was a FEI (ThermoFisher Inc., Hilsboro, OR) Quanta 250FEG Environmental SEM. For cryo-TEM analysis the mixture was rapidly frozen in liquid ethane. Detailed characterization was performed using a JEOL (JEOL USA Inc., Peabody, MA) GrandARMTM 300F probe corrected TEM operated at $300 \mathrm{kV}$. Cryo-analysis was carried out in a Gatan liquid nitrogen cryo-transfer holder, and Serial EM was used to control electron dose. All the equipment is part of the Radiochemical Microscopy Suite in the Radiochemical Processing Laboratory at PNNL.

The initial stages of the $\mathrm{U}$ oxalate formation are displayed in Figure 1. The reaction was halted by vitrifying the reactants on a TEM grid. This way, the early, meta-stable stages of particles could be investigated. Precipitate sizes and morphologies can be analyzed as a function of reaction time. Figure 2 shows STEM-EELS images acquired from a non-cryo STEM-EELS. Images after $10 \mathrm{sec}$ show the larger particles consisting of agglomerates of smaller particles grown by a particle attachment process. Cryo-TEM will show the intermediate stages of this non-classical growth process. [5]
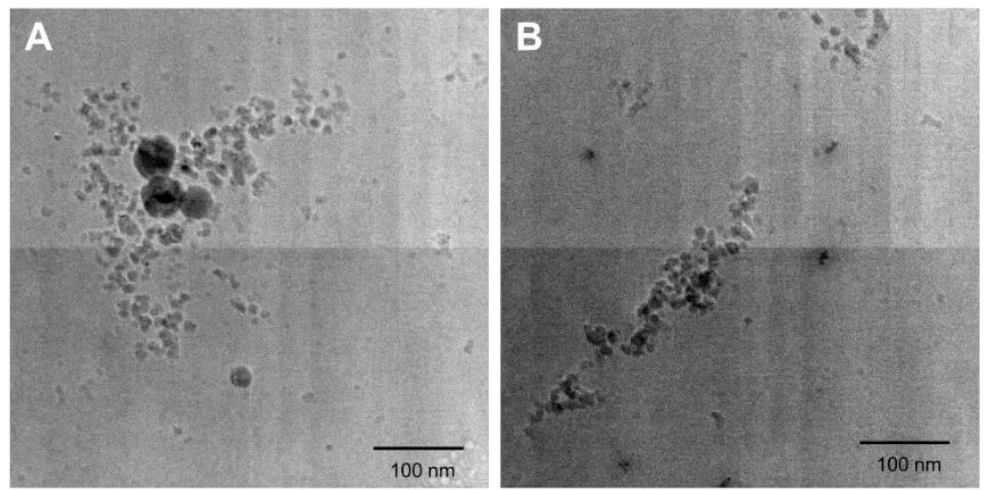

Figure 1. (A and B) CryoEM TEM images of U(IV) oxalate showing the distribution of nanoparticles that form the larger observable structures. The line across the middle is an artifact from the camera system. 

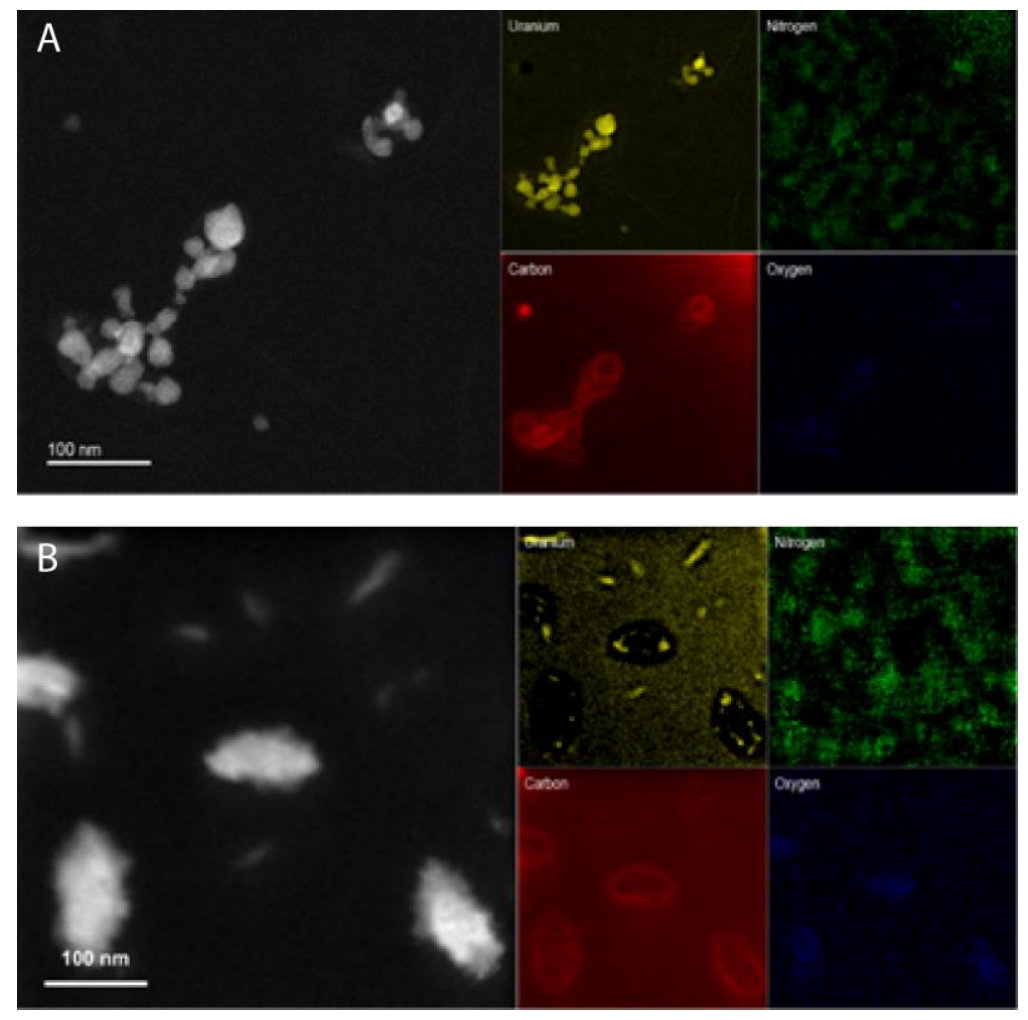

Figure 2. STEM-EELS of U(IV) Oxalate formation at (A) 0 secs and (B) 10 secs. The images show a high magnification view and elemental mapping with EELS showing uranium distribution in the particle agglomerates. The larger particles in the (B) are agglomerates of smaller ones.

\section{References}

[1] W. Runde, L. F. Brodnax, G. Goff, A. C. Bean, and B. L. Scott, "Directed Synthesis of Crystalline Plutonium(III) and (IV) Oxalates: Accessing Redox-Controlled Separations in Acidic Solutions," Inorganic Chemistry, vol. 48, no. 13, pp. 5967-5972, 2009/07/06 2009, doi: 10.1021/ic900344u.

[2] V. Tyrpekl, M. Beliš, T. Wangle, J. Vleugels, and M. Verwerft, "Alterations of thorium oxalate morphology by changing elementary precipitation conditions," Journal of Nuclear Materials, vol. 493, pp. 255-263, 9// 2017, doi: https://doi.org/10.1016/j.jnucmat.2017.06.027.

[3] J. J. De Yoreo et al., "Crystallization by particle attachment in synthetic, biogenic, and geologic environments," Science, vol. 349, no. 6247, 2015-07-31 00:00:00 2015, doi: 10.1126/science.aaa6760.

[4] J. A. Soltis, W. C. Isley, M. Conroy, S. M. Kathmann, E. C. Buck, and G. J. Lumetta, "In situ microscopy across scales for the characterization of crystal growth mechanisms: the case of europium oxalate," CrystEngComm, 10.1039/C7CE01450C vol. 20, no. 20, pp. 2822-2833, 2018, doi: 10.1039/C7CE01450C.

[5] Work supported by the Department of Homeland Security. PNNL is operated for the U.S. Department of Energy (DOE) by Battelle Memorial Institute under Contract DE-AC06-76RL0 1830. 